\title{
Quality changes and antioxidant properties of microencapsulated kenaf (Hibiscus cannabinus L.) seed oil during accelerated storage.
}

\begin{abstract}
Quality changes and antioxidant properties of microencapsulated kenaf (Hibiscus cannabinus L.) seed oil (MKSO) were investigated at 6-day intervals during 24 days of storage at $65{ }^{\circ} \mathrm{C}$. DPPH, ABTS, FRAP and BCB assays were used to determine the antioxidant activity (AOA) and their correlations were reported. Total flavonoids content (TFC) and oxidative stability in terms of evolution of thiobarbituric acid reactive substances (TBARS) were also studied. The results showed that there was a significant $(\mathrm{p}<0.05)$ effect of microencapsulation whereby AOA of MKSO showed only a $23.28 \%$ decrease as compared to a $61.51 \%$ decrease in the control bulk oil (KSO) when assessed by FRAP. MKSO showed significantly ( $\mathrm{p}<0.05$ ) lower reduction of its initial TFC as compared to KSO and total increase in TBARS of MKSO was reported to be significantly $(\mathrm{p}<0.05)$ lower than that of KSO. AOA determined by all assays were well correlated with TFC and also among themselves. Microencapsulation of kenaf seed oil was found to be effective in the stabilisation of natural antioxidants and prolonging the shelf-life.
\end{abstract}

Keyword: 2,2-Diphenyl-1-picrylhydrazyl (DPPH·) radical scavenging activity assay; 2,2'Azino-bis(3-ethylbenzothiazoline-6-sulphonic acid) (ABTS·+) radical scavenging assay; Ferric reducing antioxidant power (FRAP); $\beta$-Carotene bleaching (BCB) assay; Total flavonoid content(TFC); Thiobarbituric acid reactive substances (TBARS) 